\title{
Uncovering deception in social media
}

\section{A special issue in Springer Journal Social Network Analysis and Mining.}

\author{
Huan Liu · Jiawei Han · Hiroshi Motoda
}

Received: 3 January 2014/Accepted: 8 January 2014/Published online: 25 February 2014

(c) Springer-Verlag Wien 2014

\section{Introduction}

Social media is quickly arising as a new, popular form of media. Facebook, Twitter, and LinkedIn are some examples of an inordinate number of social media services that are loved and used by people of all walks of life for various purposes such as sharing news, expressing opinions, documenting thoughts, launching political campaigns, maintaining and developing friendships or professional connection. Some key characteristics of social media include low entry barrier, instant updates (thus, instant gratification), large numbers of friends, open platform, and anonymity. The last two properties make people comfortable to become users but also make social media vulnerable to activities of ill intentions. Deception in social media is an epitome of such activities. Deception is a distortion with an intention to mislead users, analysts, organizations, etc. A distortion can be about content, source, identity, age, sex, or location, among many. Deception is encouraged or made easy by the unique circumstances of social media. Deception is rampant in social media for a wide range of reasons, being an innocent white lie or resulting in a dire consequence where one's job or life is at stake. Hence, it is important to research deception

This article is part of the Topical Collection on Uncovering Deception in Social Media.

\section{H. Liu $(\bowtie)$}

Arizona State University, Tempe, Arizona, USA

e-mail: Huan.Liu@asu.edu

J. Han

University Illinois at Urbana-Champaign, Champaign, Illinois, USA

H. Motoda

Osaka University, Osaka, Japan in social media. It is necessary to uncover deception in social media so that we can improve our awareness and confidence in using social media, design and develop countermeasures to "separate wheat from chaff" to minimize negative impact of deception.

This special issue aims to identify issues of deception in social media, review the state-of-the-art research on deception, offer a convenient venue for sharing multi-disciplinary research findings, and identifying pressing issues and future challenges. It is a truly little treaded and relatively new area that crosses different disciplines. Thus, the process of completing this special issue is unusually timeconsuming and labor-intensive: soliciting submissions, enlisting expert reviewers, chasing reviews, revising and re-reviewing, and discussion of paper relevance and selection. With the support of the authors interested in this special issue, altruistic efforts of expert reviewers worldwide, and assistance of the journal office, we are pleased to present three articles that deliberate various issues of uncovering deception.

The first article, "Deception detection: dependable or defective?", starts reviewing existing research in deception, in search of answers to intuitive questions such as "how do human beings tell the differences between truths and lies and void being deceived? And is it possible for a machine to determine the veracity of any given statement or set of statements ...?" Among definitions of deception, the authors settle on a definition that leads with intent, and categorize lies as a specialization of deception. They present a comprehensive and concise review on deception detection cues and some empirical results on the effectiveness of these cues. After they elaborate the notion of deception and the relationship of social, psychological, and technical views and point out some research gaps, the authors show deception detection applications in 
authorship identification, plagiarism finding, and sexual predator detection. This article serves a good introduction to the existing work in some related disciplines, learning about some limited successes in real-world applications.

The second article, "Tweet, but verify: epistemic study of information verification on twitter", approaches the problem of uncovering deception in tweets via epistemology. It is a branch of psychology studying what are the factors that make humans trust some piece of information. Information verification on Twitter is a challenging problem. As the authors point out, information about current events often appears on Twitter much earlier than it is reported in traditional news media. Applying epistemology, the authors aim to find out how the impression on different features of a tweet can help or confound its veracity assessment. Epistemology of tweets suggests four features such as authority, plausibility and support, independent corroboration, and presentation. They then analyze the accuracy of credibility perceptions on different features of witness pictures posted on Twitter during Hurricane Sandy. The credibility ratings are obtained by Amazon's Mechanical Turk. The study reports interesting findings about features in their positive and negative roles in information verification, and additional factors that affect users' perceptions with some open problems.

The third article, "Why and how to deceive: game and complexity results with sociological evidence", takes a game theoretical modeling approach to the study of deception in social media, investigating when, how, and why users deceive. The authors consider deception as an intentional form of abusive behavior violating social norms and rules of use of social networking sites. They address a modeling challenge when modelers have little reliable data on deceptive activities on social networking sites. They try to ground their game theoretic model on data. One dataset is a web-based survey with three aspects of user behavior: privacy awareness, attitude toward information withholding and practice, and attitude toward lies and misrepresentation. The other dataset is the data collected from a forum where the ground-truth information about abusive behavior is given. It is interesting to see how the authors use data to infer model parameters and then employ the model to analyze and illustrate social behavior with respect to deception.

The three articles in this special issue are some glimpse of latest research work on uncovering deception. A common feature of the three articles is that all provide an informative literature survey related to their work. It is highly valuable and effective for a reader to quickly get familiar with the literature of excellent variety. The first two articles focus on how deception can be detected from a user's perspective and are representative of the approaches basing mostly on single short text or image analysis to uncover deceptive activities. They have not discussed the role of social network analysis in uncovering deception. However, in the third article's related work, the authors mention some projects that employ social network analysis for uncovering deception. Its conclusion is that it is more difficult to deceive in a networked environment. A new trend, however, deviates from this observation. As a chief goal of social media is social networking, deceptive users nowadays adapt fast and are creative in building fictitious small networks and grow them by befriending real and honest users. The third article takes a game-theoretic modeling approach to understanding and draw insights on how and why deceptive users choose to deceive. Its findings should help develop better methods of uncovering deception in social media. Selecting this set of articles from a much larger number of submissions is not an easy task. We had opportunities to read submissions that are related to deception research, but not pertinent to this special issue. This slim special issue, in a way, suggests that the research of deception in social media is still in its infancy. We sincerely hope a future issue of this sort will be able to bring the readers many papers on this emerging and challenging topic, which is indeed one of our original motivations of organizing this special issue. 